\title{
PHƯƠNG PHÁP XÁC ĐỊNH TỌA ĐỘ TRẠM CORS THEO KHUNG THAM CHIỂU MẠTT ĐẤT QUỐC TẾ - ITRF BĂNG BERNESE 5.0
}

\author{
PHẠM CÔNG KHẢI, NGUYẼ̃N VIÉT NGHĨA \\ Trường Đại học Mỏ - Địa chất
}

\section{Tóm tắt:}

Hiện nay trên thế giới đã có nhiều quốc gia và khu vực đã cho xây dựng hệ thống trạm CORS với những trình độ kỹ thuật và ứng dụng khác nhau. Ở Việt Nam hệ thống trạm CORS đã bắt đầu được đưa vào ứng dụng thư nghiệm. Được sự tài trợ của tập đoàn máy trắc địa Nam Phương (SOUTH) đã lắp đặt tại trường Đại học Mỏ-Địa chất một trạm CORS.

Để hệ thống hoạt động được cần phải xác định tọa độ chính xác của trạm CORS trong hệ WGS84 và do đó cần thiết phải đo nối đến trạm quan trắc quốc tế IGS.

Bài báo này trình bày phương pháp xử lý số liệu và xác định tọa độ cho trạm CORS lắp đặt ở Trường Đại học Mỏ - Địa chất với thời gian thu số liệu liên tục trong 18 ngày bằng phần mềm Bernese 5.0 .

\section{1. Đặt vấn đề}

Hệ thống trạm tham chiếu liên tục (CORS) được định nghĩa là một hoặc nhiều trạm tham chiếu GNSS vận hành liên tục cố định, kết hợp ứng dụng công nghệ máy tính hiện đại và hệ thống mạng internet truyền dữ liệu tạo thành một mạng lưới đồng bộ. Trên cơ sở vận hành của trạm CORS, có thể sử dụng phương pháp đo động xử lý tức thời RTK và chỉ cần sử dụng thêm 1 máy thu di động GNSS có khả năng kết nối dữ liệu qua GPRS (viễn thông) tới trạm CORS thì có thể tiến hành xác định tọa độ điểm máy thu di động chính xác, nhanh chóng với khoảng cách tăng đáng kể.

Để có thể vận hành và đưa vào phục vụ, vấn đề đặt ra là cần phải tiến hành xác định được tọa độ tuyệt đối có độ chính xác cao cho trạm CORS trong hệ tọa độ WGS84, bằng cách kết nối đến trạm quan trắc IGS quốc tế kết hợp sử dụng chương trình xử lý dữ liệu đo GNSS độ chính xác cao như Bernese, GAMIT/GLOBK, v.v... Như đã biết, từ năm 1995 sau khi xác định lại tọa độ các trạm quan sát độ lệch giữa WGS-84 và ITRF đã xác định được độ lệch cỡ 10 centimet [4].

Với sự hợp tác của tập đoàn South (Trung Quốc) và Khoa Trắc địa trường Đại học Mỏ - Địa chất, đã tiến hành lắp đặt một trạm CORS có tên trạm N001.

Bài báo trình bày kết quả xác định tọa độ điểm trạm CORS-N001 trong hệ tọa độ quốc tế WGS-84 theo khung quy chiếu trái đất quốc tế ITRF-2008 bằng phần mềm Bernese 5.0.

\section{Hệ thống trạm CORS trường Đại học Mỏ - Địa chất}

Hệ thống trạm CORS tại trường Đại học Mỏ - Địa chất, được đặt tại nhà $B$ của trường. (hình 1). 


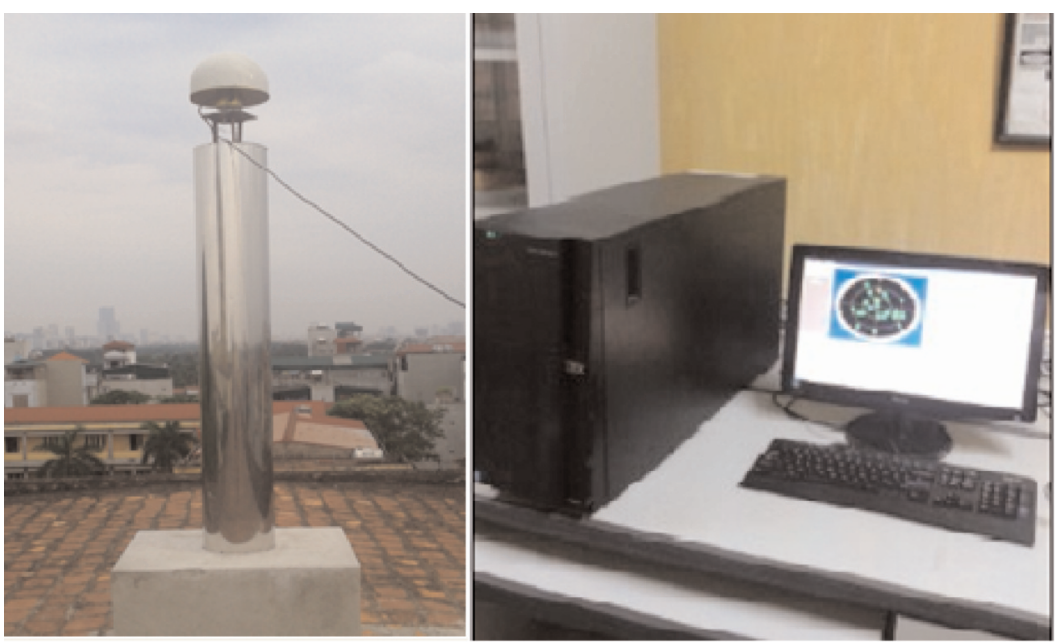

Hình 1: Trạm CORS - N001 tại trường Đại học Mỏ - Địa chất

Hệ thống trạm CORS này bao gồm các bộ phận:

\section{a. Bộ phận trạm thu chuẩn NRS}

Trạm thu được đặt ở nơi an toàn, thông thoáng, ít bị ảnh hưởng của hiệu ứng đa đường dẫn, lưới điện ổn định. Hệ thống máy thu là loại NRS NET S8, sử dụng bo mạch chủ của Trimble BD970, 220 kênh, hỗ trợ thu tín hiệu vệ tinh GPS, Glonass, Bắc Đẩu ; hỗ trợ GIOVE - A; GIOVE - B, tần suất thu thập có thể đạt 50 Hertz. Hệ thống có cổng kết nối đa năng, có khả năng phục hồi sau khi mất điện và tự động hoạt động liên tục như cài đặt ban đầu; trường hợp sau khi bị mất mạng internet vẫn bảo lưu số liệu và sau khi kết nối được với mạng thì số liệu tiếp tục tự động trút vào máy. (hình 2a). [ ]

Ăng-ten thu sử dụng vật liệu ít co dãn, có khả năng loại bỏ sóng tạp do hiệu ứng đa đường dẫn, thiết kế định tâm với độ ổn định của tâm ăng-ten đạt đến $0,8 \mathrm{~mm}$ và có khả năng thu tín hiệu ở góc ngưỡng rất nhỏ. (hình 2b)

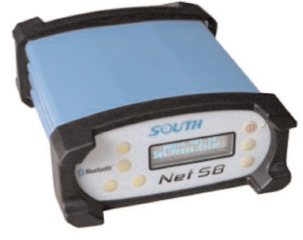

(a)

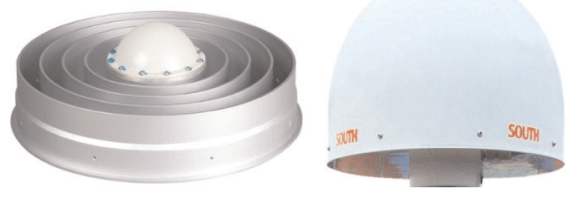

(b)

Hình 2: (a) Trạm thu chuẩn NRS NET S8, (b) Antenna của hãng South - Trung Quốc.

\section{b. Trung tâm điều khiển hệ thống NRS}

Trung tâm xử lý số liệu, thông qua máy tính chủ (Server), có thể phân cấp quản lý, tùy theo từng đối tượng người sử dụng bằng hai phần mềm đi kèm: NRS-Center (phục vụ tính toán số liệu, phân bổ số liệu trạm thu tĩnh) và NRS-Server nhận và gửi thông tin sai phân cho các máy thu GNSS di động. Tiến hành xử lý số liệu của mạng lưới đo động RTK, đồng thời hiệu chỉnh các số nguyên đa trị của toàn mạng, thiết lập mô hình cải chính (gồm cải chính sai số tầng đối lưu, tầng điện ly, quỹ đạo vệ tinh). 


\section{c. Phần mềm Bernese 5.0}

Phần mềm Bernese do Viện Thiên Văn - Trường Đại học Bern - Thụy Sỹ xây dựng với các phiên bản 4.0 (năm 1996), 4.2 (năm 1999), 5.0 (năm 2004) và phiên bản mới nhất 5.2 (năm 2013). Phần mềm Bernese được công nhận là phần mềm đa năng có độ chính xác cao. Đã có rất nhiều trường đại học viện nghiên cứu sử dụng với nhiều mục đích khác nhau nhu:

- Xử lý nhanh lưới quy mô nhỏ

- Xử lý mạng lưới tự động

- Giải số nguyên đa trị cho các cạnh dài (trên 2000 km)

- Thành lập mô hình tầng đối lưu, tầng điện ly

- Kết hợp xử lý số liệu GPS và GLONASS với nhiều loại máy thu

- Tính toán, xác định độ lệch đồng hồ và chuyển đổi thời gian

- Xác định quỹ đạo và các tham số Trái đất.

Bernese thường xuyên cập nhật những kết quả mới nhất về: tham số quay của Trái đất, mô hình chuyển động mảng toàn cầu, v.v... và cho phép lựa chọn, can thiệp để xử lý cho ra số liệu tốt nhất. Menu của chương trình có khoảng gần 100 chương trình, phân thành các thành phần chính: trình

- Phần chuyển đổi: Chuyển đổi các loại dữ liệu định dạng Rinex về cầu trúc của chương

- Chiết xuất dữ liệu

- Quỹ đạo: xử lý các thông tin quỹ đạo vệ tinh

- Đồng bộ đồng hồ máy thu và đồng hồ vệ tinh

- Xử lý: gồm môdul GPSEST và môdul ADDNEQ2: xử lý, kiểm tra, bình sai, ước tính các tham số, xác định các hệ phương trình chuẩn. Tùy theo mục đích có thể xử dụng các phương trình sai phân bậc 1 đến bậc 5 , tổ hợp bậc 1 và 2 , tổ hợp bậc 3 và 4 , DTEC, MELWUEBB.

- Mô phỏng

- Dịch vụ: So sánh tọa độ giữa các ca đo, ngày đo, tính chuyển tọa độ, ...

- Xử lý tự động (BPE): Tự động xử lý, tính toán các số liệu GNSS

\section{Xác định tọa độ trạm CORS tại Trưò̀ng ĐH Mỏ - Địa chất bằng Bernese 5.0}

Để xác định tọa độ cho điểm trạm CORS - N001 trong hệ tọa độ WGS-84, tiến hành kết nối với 11 điểm IGS quốc tế (BJFS - Bắc Kinh; SHAO - Thượng Hải; WUHN - Vũ Hán; LHAZ - Tây Tạng - Trung quốc; DAEJ - Hàn Quốc; PIMO - Philippine; NTUS - Singapore; TCMS, TNML, TWTF - Đài Loan; IISC, - Ân Độ) và được tính trong khung quy chiếu trái đất ITRF 2008 (hình 3) bằng chương trình xử lý dữ liệu đo GNSS độ chính xác cao Bernese 5.0. Trong đó, các điểm BJFS, NTUS, WUHN sử dụng số liệu thu của GPS và GLONASS. 


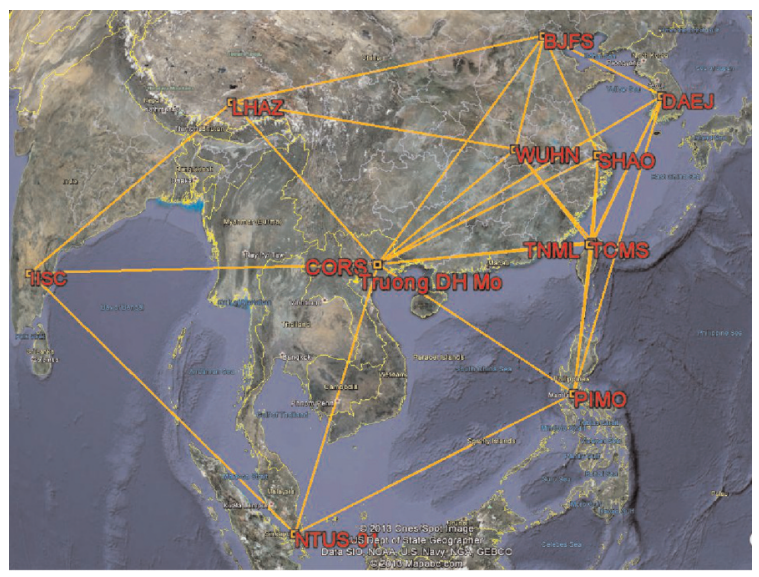

Hinh 3: So đồ điểm trạm CORS kết nối với các điểm IGS

Số liệu trạm CORS được kết nối tới các điểm IGS từ ngày 24 tháng 5 năm 2013 đến 19 tháng 6 năm 2013 có dạng Rinex. Tổng cộng số ngày kết nối là 18 ngày vì có 6 ngày không kết nối là các ngày 25,31 tháng $5 ; 1,9,15,16$ tháng 6 .

Thông qua địa chỉ trang web http://holt.oso. chalmers.se/loading/ có thể cập nhật các thông số cải chính thủy triều đại dương cho các trạm và cập nhật các số hiệu chỉnh tầng điện ly, dcb ở trang web của Bernese.

Thông qua môdul CODSPP, tiến hành công tác đồng bộ đồng hồ máy thu và đồng hồ vệ tinh. Quá trình xử lý này hoàn toàn xử lý trên tín hiệu đo code. Sau bước đồng bộ đồng hồ máy thu, tín hiệu đo code sẽ không được đề cập đến nữa, các xử lý sau này đều là xử lý tín hiệu đo phase.

Để giải cạnh, tiến hành sử dụng môdul GPSEST để giải cạnh sơ bộ bằng hàm sai phân bậc 3 (L3) với tất cả các trị đo và sử dụng hàm ánh xạ cho thành phẩn ướt của độ trễ do tầng đối lưu gây ra (mô hình ẩm Niell) với khoảng dãn cách 4 giờ. (hình 4) và tiến hành lọc bỏ các trị đo dư thô bằng môdul RESRMS và SATMRK. Sao cho các giá trị sai số trọng số đơn vị trước bình sai khi giải lại bằng môdul GPSEST nhỏ hơn 0.0015 thì khi đó cạnh được giải đảm bảo chất lượng.
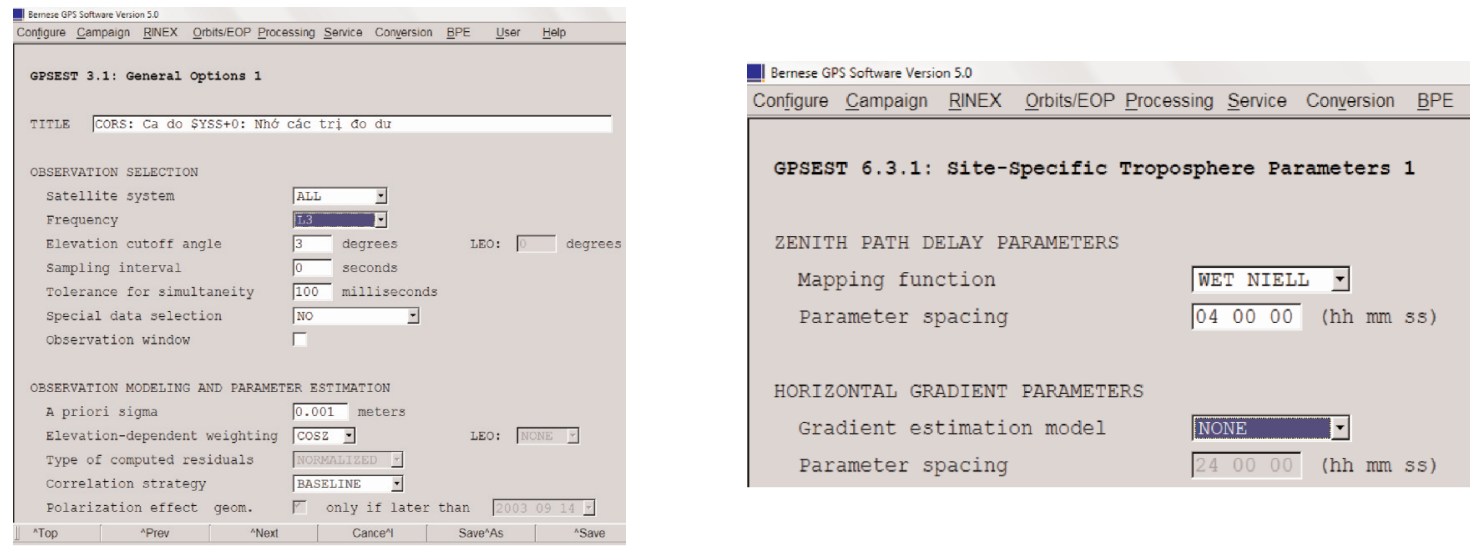

Hình 4: Tham số cài đặt trong môdul GPSEST để ghi nhớ trị đo du 
Tiếp theo, sử dụng môdul GPSEST tiến hành bình sai giải cạnh lần 2 và lần 3 với khoảng thời gian giãn cách lần lượt 30 giây và 180 giây. Trong đó, giải cạnh lần 3 , sử dụng tham số trạm liên quan đến giải các số trị nguyên đa trị (Ambiguities), sử dụng hàm mô hình ẩm Niell với khoảng dãn cách 1 giờ và mô hình Gradient (Gradient estimation model) với khoảng thời gian 24 giờ. (hình 5).
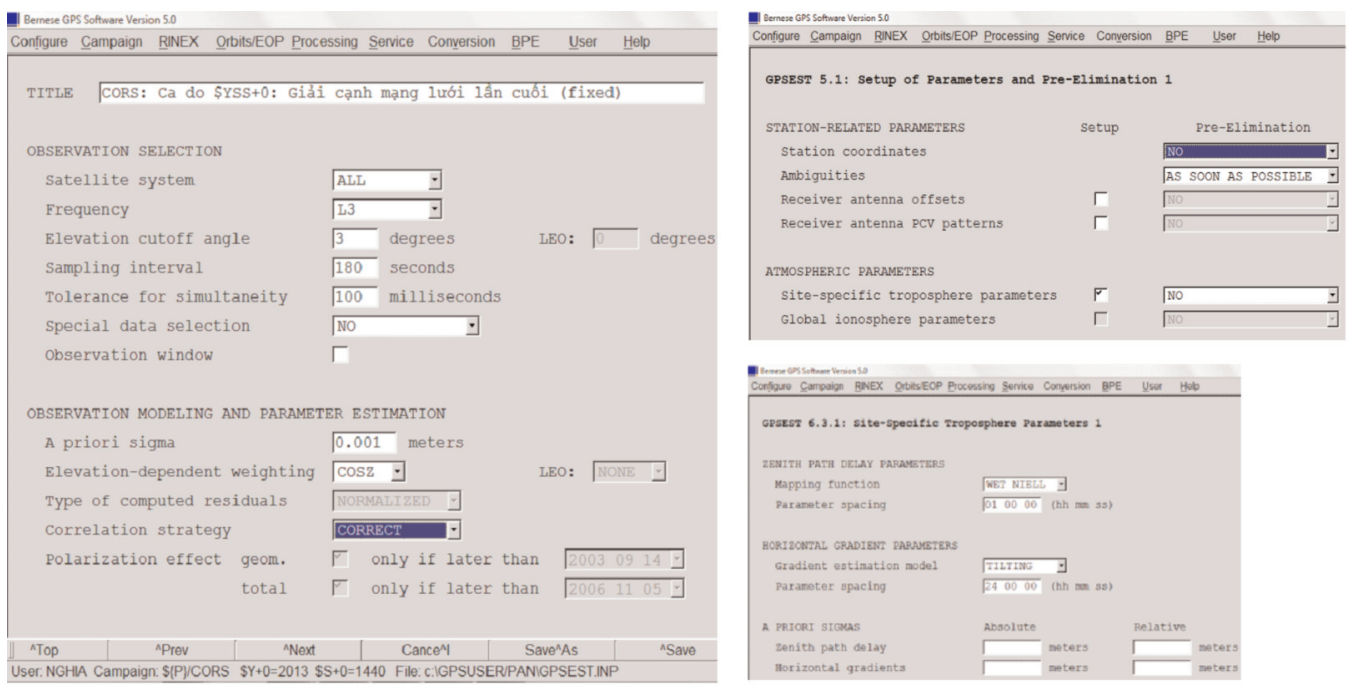

Hình 5: Tham số cài đặt trong môdul GPSEST để giải cạnh lần 3

Sau đó, sử dụng môdul ADDNEQ2, để tiến hành liên kết kiểm tra chặt chẽ dữ liệu tọa độ điểm vừa tính được với tọa độ các điểm IGS trong khung quy chiếu trái đất ITRF-2008 và kết quả tính được xuất ra định dạng phương trình chuẩn của phần mềm (Normal equation) dạng *.NQ0 cho từng ngày đo. Tiếp theo, so sánh độc lập Helmert để xác định tọa độ với việc sử dụng tọa độ và vận tốc của các điểm ITRF-2008 làm điểm cơ sở để tính thành phần tọa độ, vận tốc chuyển dịch và sai số của các điểm trong cùng một hệ thống tọa độ không gian địa tâm $(X, Y, Z)$, địa lý $(N, E, U)$ cho 1 ngày cụ thể.

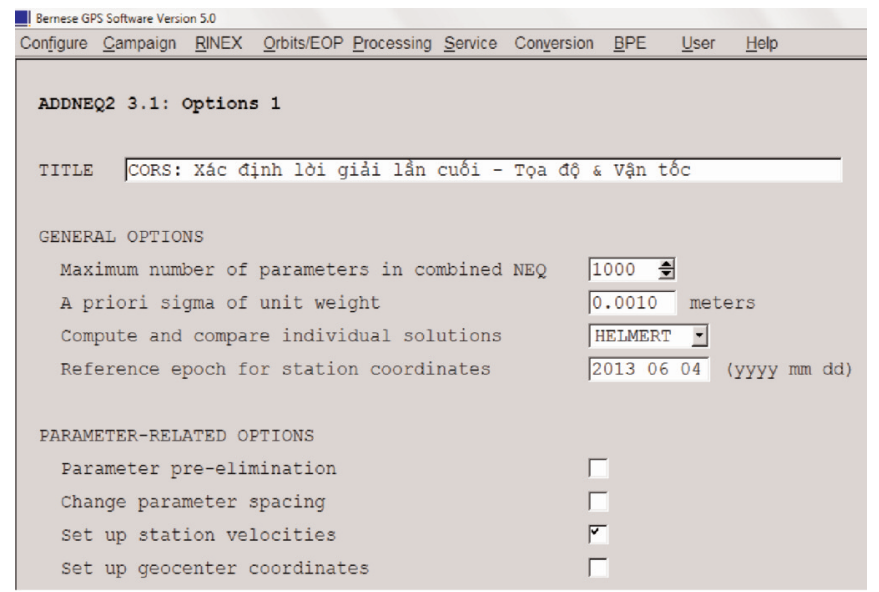

Hình 6: Sử dụng giải pháp tính và so sánh Helmert để xác định tọa độ và vận tốc 
Kết quả sau khi tính toán và so sánh được tổng hợp và trình này ở bảng 1 . Ở đây, các điểm chưa cần quan tâm đến vận tốc chuyển dịch của điểm trạm CORS - N001 nên tác giả không trình bày, mà chỉ trình bày các thành phần tọa độ cùng sai số tương ứng.

Bảng 1: Thành phần tọa độ và sai số trong hệ tọa độ WGS-84

(thời điểm 1 giờ 02 phút 30 giây, ngày 04 tháng 6 năm 2013)

\begin{tabular}{|c|c|c|c|c|c|c|}
\hline Tên điểm & $\begin{array}{l}\text { Thành phần } \\
\text { tọa độ }\end{array}$ & $\begin{array}{c}\text { Giá trị tọa độ sau } \\
\text { bình sai }\end{array}$ & Sai số RMS & $\begin{array}{l}\text { Thành phần } \\
\text { tọa độ }\end{array}$ & $\begin{array}{c}\text { Giá trị tọa độ sau } \\
\text { bình sai }\end{array}$ & Sai số RMS \\
\hline \multirow{3}{*}{ N001 } & $\mathrm{E}$ & 1054624.547827 & 0.0001 & $\mathrm{X}$ & -1618564.5576 & 0.0002 \\
\hline & $\mathrm{N}$ & $\begin{array}{llll}21 & 4 & 17.959085\end{array}$ & 0.0001 & $\overline{\mathrm{Y}}$ & 5730005.6206 & 0.0004 \\
\hline & $\bar{U}$ & 0.9251 & 0.0005 & $Z$ & 2278800.1280 & 0.0002 \\
\hline \multirow{3}{*}{ BJFS } & $\bar{E}$ & 1155332.957690 & 0.0001 & $\mathrm{X}$ & -2148744.3455 & 0.0002 \\
\hline & $\mathrm{N}$ & 393630.961952 & 0.0002 & $\mathrm{Y}$ & 4426641.2277 & 0.0005 \\
\hline & $\bar{U}$ & 87.4730 & 0.0006 & Z & 4044655.8698 & 0.0003 \\
\hline \multirow{3}{*}{ DAEJ } & $\mathrm{E}$ & 1272228.135474 & 0.0002 & $\mathrm{X}$ & -3120042.2172 & 0.0003 \\
\hline & $\mathrm{N}$ & 362357.940457 & 0.0001 & $\bar{Y}$ & 4084614.7731 & 0.0005 \\
\hline & $\bar{U}$ & 116.8446 & 0.0006 & $Z$ & 3764026.8438 & 0.0004 \\
\hline \multirow{3}{*}{ HSC } & $E$ & 773413.364681 & 0.0004 & $\mathrm{X}$ & 1337936.1054 & 0.0003 \\
\hline & $\mathrm{N}$ & 13116.206923 & 0.0002 & $\bar{Y}$ & 6070317.1240 & 0.0014 \\
\hline & $\bar{U}$ & 843.7052 & 0.0014 & Z & 1427877.0782 & 0.0004 \\
\hline \multirow{3}{*}{ LHAZ } & $E$ & 91614.505680 & 0.0002 & $\mathrm{X}$ & -106941.8237 & 0.0002 \\
\hline & $\mathrm{N}$ & 293926.399411 & 0.0001 & $\bar{Y}$ & 5549269.8253 & 0.0007 \\
\hline & $\bar{U}$ & 3624.6167 & 0.0008 & $\bar{Z}$ & 3139215.1274 & 0.0003 \\
\hline \multirow{3}{*}{ NTUS } & $\bar{E}$ & 1034047.850999 & 0.0001 & $\mathrm{X}$ & -1508023.0421 & 0.0002 \\
\hline & $\mathrm{N}$ & 122044.884523 & 0.0002 & $\mathrm{Y}$ & 6195576.5830 & 0.0007 \\
\hline & $\bar{U}$ & 75.3797 & 0.0008 & $Z$ & 148799.3612 & 0.0002 \\
\hline \multirow{3}{*}{ PIMO } & $E$ & 121439.825827 & 0.0002 & $\bar{X}$ & -3186293.6975 & 0.0004 \\
\hline & $\mathrm{N}$ & 14388.593138 & 0.0002 & $\bar{Y}$ & 5286624.3462 & 0.0006 \\
\hline & $\bar{U}$ & 95.5476 & 0.0007 & $Z$ & 1601158.3573 & 0.0002 \\
\hline \multirow{3}{*}{ SHAO } & $\bar{E}$ & 121121.609450 & 0.0002 & $\bar{X}$ & -2831733.7544 & 0.0002 \\
\hline & $\mathrm{N}$ & 31558.709684 & 0.0001 & $\bar{Y}$ & 4675665.8461 & 0.0005 \\
\hline & $\bar{U}$ & 22.0423 & 0.0006 & $Z$ & 3275369.3288 & 0.0003 \\
\hline \multirow{3}{*}{ TCMS } & $E$ & $\begin{array}{|lll|}120 & 59 & 14.613392 \\
\end{array}$ & 0.0002 & $\mathrm{x}$ & -2982783.1668 & 0.0003 \\
\hline & $\mathrm{N}$ & $24 \quad 4752.747215$ & 0.0001 & $\bar{Y}$ & 4966659.9928 & 0.0005 \\
\hline & $\bar{U}$ & 77.2438 & 0.0006 & $Z$ & 2658809.3788 & 0.0003 \\
\hline \multirow{3}{*}{ TNML } & $\bar{E}$ & 1205914.450511 & 0.0002 & $\mathrm{X}$ & -2982779.3508 & 0.0003 \\
\hline & $\mathrm{N}$ & $24 \quad 4752.634285$ & 0.0001 & $\mathrm{Y}$ & 4966662.5248 & 0.0006 \\
\hline & $\bar{U}$ & 75.8653 & 0.0007 & Z & 2658805.6462 & 0.0003 \\
\hline \multirow{3}{*}{ TWTF } & $E$ & 121952.212365 & 0.0002 & $\bar{X}$ & -2994428.4187 & 0.0003 \\
\hline & $\mathrm{N}$ & 245712.830503 & 0.0001 & $\bar{Y}$ & 4951309.1839 & 0.0005 \\
\hline & $\bar{U}$ & 201.5373 & 0.0006 & $Z$ & 2674496.7141 & 0.0003 \\
\hline \multirow{3}{*}{ WUHN } & $\bar{E}$ & 1142126.153686 & 0.0002 & $\bar{X}$ & -2267749.6867 & 0.0003 \\
\hline & $\bar{N}$ & 303153.946953 & 0.0001 & $\bar{Y}$ & 5009154.2159 & 0.0007 \\
\hline & $\bar{U}$ & 25.8540 & 0.0008 & $\bar{Z}$ & 3221290.6190 & 0.0004 \\
\hline
\end{tabular}


Ở đây, các giá trị "Sai số RMS" trong bảng 1 là các sai số tương đối, không phản ánh sai số thực của các thành phần tọa độ điểm. Để xác độ ổn định các thành phần sai số điểm, trong mốdul $A D D N E Q 2$ có trình bày chi tiết sai số các ngày đo và sai số trung bình của thành phần tọa độ với phương pháp so sánh độc lập.

Bảng 2: Giá trị sai số thành phần các điểm đo với giải pháp so sánh độc lập (mm)

\begin{tabular}{|c|c|c|c|c|}
\hline & Trạm & N & E & U \\
\hline 1 & BJFS & 0.87 & 1.53 & 5.95 \\
\hline 2 & DAEJ & 2.09 & 2.32 & 5.02 \\
\hline 3 & HSC & 2.01 & 3.06 & 4.01 \\
\hline 4 & LHAZ & 1.44 & 1.58 & 4.89 \\
\hline 5 & N001 & 2.00 & 1.36 & 4.67 \\
\hline 6 & NTUS & 1.42 & 1.71 & 4.85 \\
\hline 7 & PIMO & 1.22 & 2.25 & 6.90 \\
\hline 8 & SHAO & 0.89 & 1.77 & 4.64 \\
\hline 9 & TCMS & 1.13 & 2.22 & 4.02 \\
\hline 10 & TNML & 1.74 & 5.36 & 8.47 \\
\hline 11 & TWTF & 1.04 & 1.46 & 3.86 \\
\hline 12 & WUHN & 1.49 & 4.02 & 6.70 \\
\hline
\end{tabular}

Đồng thời trong kết quả bình sai giải cạnh cuối cùng được tính bằng môdul ADDNEQ2 có trình bày sai số (RMS) của tham số chuyển đổi Helmert với lời giải kết hợp được thể hiện ở hình 7.

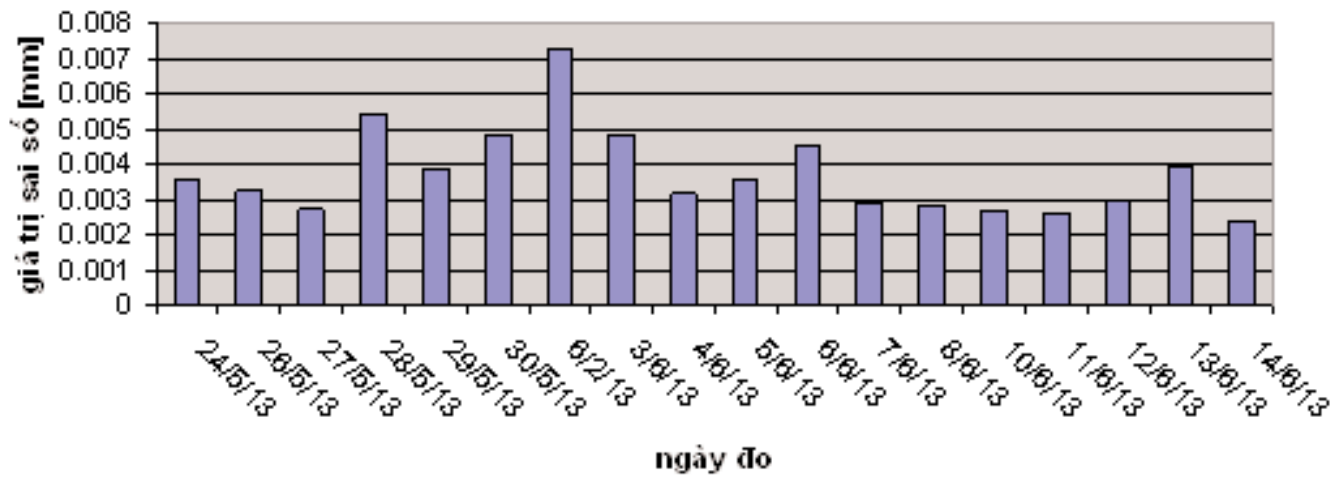

Hình 7: Sai số (RMS) của tham số chuyển đổi Helmert với lời giải kết hợp

\section{Kết luận}

Sau 18 ngày đo kết nối với 11 điểm IGS, tọa độ điểm trạm CORS (N001) tại Trường đại học Mỏ - Địa chất được tính bằng phần mềm Bernese 5.0 đã xác định được tọa độ của điểm N001 trong hệ tọa độ WGS-84 theo khung quy chiếu quốc tế ITRF-2008 ở ngày 4 tháng 6 năm 2013, với sai số tương đối và sai số tham số chuyển đổi Helmet với lời giải kết hợp tính trong 18 ngày.

(Xem tiếp trang 57) 